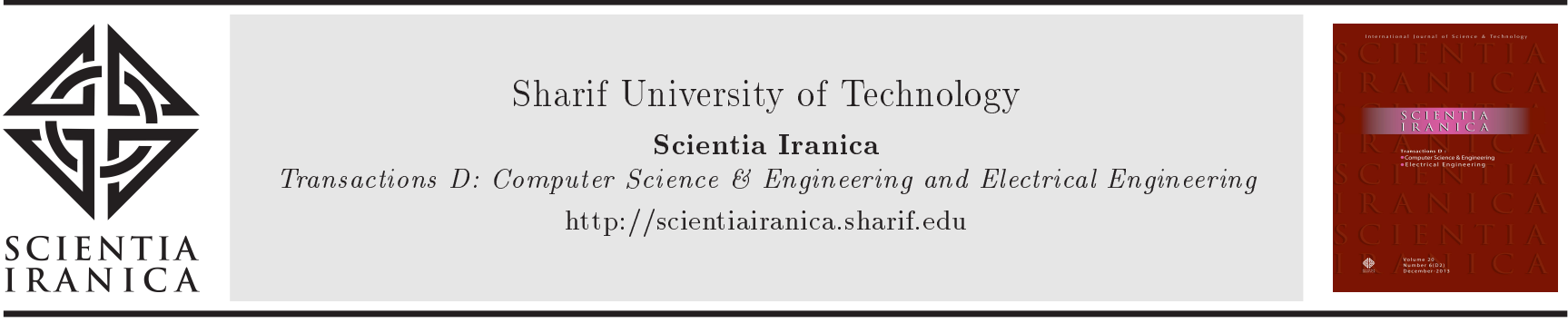

\title{
Terahertz linear to circular polarization converter based on reflective metasurface
}

\author{
M. Barkabian ${ }^{\mathrm{a}}$, N. Dalvand ${ }^{\mathrm{b}}$, H. Zandi ${ }^{\mathrm{c}, *}$, and N. Granpayeh ${ }^{\mathrm{a}}$ \\ a. Centre of Excellence in Electromagnetics, Optical Communication Lab, Faculty of Electrical Engineering, K. N. Toosi University \\ of Technology, Tehran, Iran. \\ b. Faculty of Electrical Engineering, K. N. Toosi University of Technology, Tehran, Iran. \\ c. Electronic Materials Laboratory, Faculty of Electrical Engineering, K. N. Toosi University of Technology, Tehran, Iran.
}

Received 31 August 2020; received in revised form 20 February 2021; accepted 24 May 2021

\author{
KEYWORDS \\ Metasurface; \\ Circular polarization; \\ Polarization \\ conversion; \\ Linear To Circular \\ Polarization \\ Conversion (LTC- \\ $\mathrm{PC})$; \\ THz devices.
}

\begin{abstract}
Metasurfaces are two-dimensional artificial structures, which have extraordinary electromagnetic properties. They have been used in a myriad of devices such as nano-antennas, cloaking coatings, imaging devices, flat lenses, and polarization converters over a wide range of frequencies. Due to high dependency of many devices on incident wave polarization, manipulating the polarization of electromagnetic waves would be useful, especially in the Terahertz ( $\mathrm{THz}$ ) regime. In this study, we propose a Linear To Circular Polarization Converter (LTC-PC) based on a THz reflective metasurface. For a TE linear polarization incident wave, this structure has two distinct bands; the first one lies in a wideband frequency range of $0.5-1.41 \mathrm{THz}$, in which the reflected wave will be a LeftHanded Circular Polarization (LHCP) with minimum efficiency of $89 \%$ and maximum efficiency of more than $95 \%$ in $80 \%$ of the bandwidth. The second band lies in the narrowband frequency range of $1.45-1.55 \mathrm{THz}$, resulting in a Right-Handed Circular Polarization (RHCP) wave with a minimum efficiency of $82 \%$. The proposed polarization converter can be used in optical communication and electronic devices.

(C) 2023 Sharif University of Technology. All rights reserved.
\end{abstract}

\section{Introduction}

Metamaterials are composed of periodic subwavelength structures some properties of which have not yet been found in natural materials [1,2]. A metasurface is a two-dimensional form of metamaterial with thin thickness and considerably less fabrication complexity. Despite having lower thickness, they have acceptable optical response in comparison with conventional metamaterials and therefore, they are a good replacement for bulky metamaterials [1,3-5]. Nowadays, metasurfaces are remarkably attractive because of their applications in sensors [6], imaging devices [7], absorp-

\footnotetext{
*. Corresponding author.

E-mail address: zandi@kntu.ac.ir (H. Zandi)
}

doi: $10.24200 /$ sci. 2021.56700 .4865 tion devices [8], and beam manipulation devices [9] such as holograms, cloaking devices, and polarization converters in the microwave, terahertz $(\mathrm{THz})$, and optical regimes. Due to low volume, low absorption, and tunable optical response of metasurfaces, they have been extensively used in Linear To Circular Polarization Converters (LTC-PC) [10,11]. For example, in [11], a linear polarization converter, which operated within a wide frequency range of 0.6 to $1.41 \mathrm{THz}$ with an efficiency higher than $88 \%$, was proposed. Gao et al. also proposed a wideband LTC-PC based on graphene metasurface. This device was tunable by changing the Fermi energy of graphene [12]. A cross-polarization convertor using metasurface was introduced in three distinct frequency bands for 9.8-12.2, 19.0-22.6, and 24.9-29.5 GHz. The Polarization Conversion Ratio (PCR) was more than 90\% [13]. Chen and Chang reported a structure composing two cascading arrays 
of complementary resonators. In this LTC-PC, the bandwidth reached two octaves in the range of 0.4$2.4 \mathrm{THz}$ [14]. An LTC-PC aperture-coupled antenna with an ultrathin single-layer metasurface was proposed in [15] to achieve boresight radiation gain of around $10 \mathrm{dBic}$ over $9.5 \mathrm{GHz}$ to $11.5 \mathrm{GHz}$. The Axial Ratio (AR) bandwidth of their proposed antenna was also $19.23 \%$. In 2020, Lin et al. proposed an ultra-wideband circular polarized maintaining reflector based on metasurface. The reflections of both Right-Handed Circular Polarization (RHCP) and LeftHanded Circular Polarization (LHCP) were attained between 8.79 and $27.09 \mathrm{GHz}$ [16]. Another polarization convertor, which was explained in [17], could convert linear polarized wave to its orthogonal polarization in two distinct frequency bands of $7.1-8 \mathrm{GHz}$ and $13.3-$ $25.8 \mathrm{GHz}$. The proposed metasurface consisted of metal patches laid diagonally on the FR4 substrate, both placed on a reflective metal sheet that enhanced the performance of the Linear Polatization Convertor (LPC) to more than $95 \% \mathrm{PCR}$.

In this work, we propose a broad-band highefficiency LTC-PC. The output wave of the structure has an AR of less than $3 \mathrm{~dB}$ and high efficiency of $95 \%$ in $80 \%$ of the bandwidth. If a linearly polarized plane wave illuminates the proposed metasurface, the reflected wave will have a wideband LHCP in the $0.5-1.41 \mathrm{THz}$ range and a narrowband RHCP in the $1.45-1.55 \mathrm{THz}$ range. The remaining of this paper is organized as follows: In Section 2, the structure design and the analysis method are described. The results are presented and discussed in Section 3. Finally, the paper is concluded in Section 4.

\section{Design and analysis method}

Figure 1 presents a three-dimensional schematic view of the $3 \times 3$ single-unit cell of our proposed LTCPC. A fully reflective gold mirror is utilized at the backplane and the substrate is polyimide with dielectric constant, loss tangent, and thickness of $3,0.001$, and $47 \mu \mathrm{m}$, respectively. As shown in Figure 1(b), the front gold pattern is composed of two crossed strips on two opposing diameter points with $200 \mathrm{~nm}$ thicknesses. Metal strips behave as inductances and the gaps between the two strips behave as the capacitor. The dimension and shape of the structure are optimized to get the widest possible bandwidth, using the Finite Element Method (FEM). As shown in Figure 1(b), the square shaped unit cell has the lattice constant (a) of $88 \mu \mathrm{m}$. The width of gold, $W$, distance between each strip and the edge of the structure, $S$, and the thickness of the substrate, $h$, are $7 \mu \mathrm{m}, 5 \mu \mathrm{m}$, and $47 \mu \mathrm{m}$, respectively. The co-polarized and cross-polarized reflection amplitudes of the proposed structure are depicted in Figure 1(c).

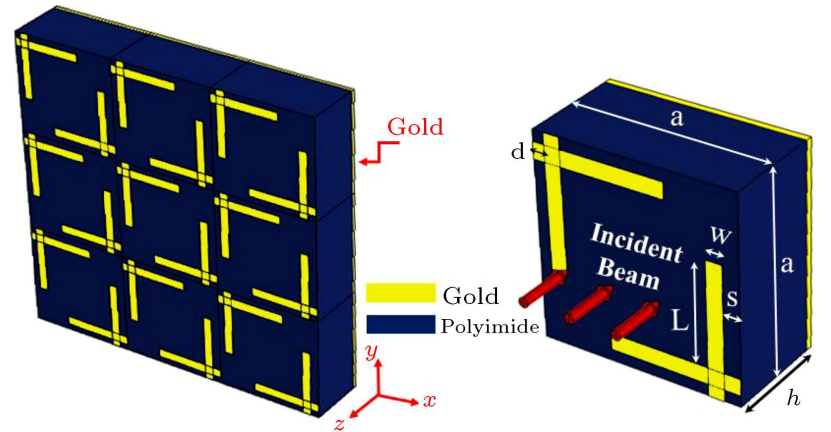

(a)

(b)

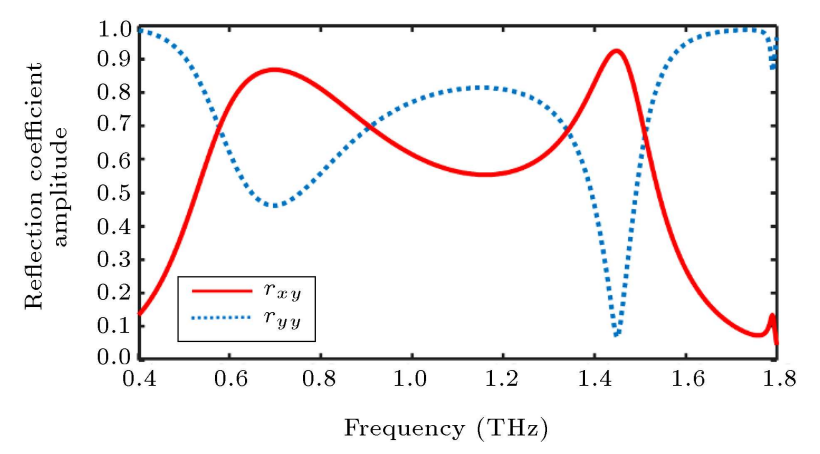

(c)

Figure 1. (a) Schematic view, (b) single unit cell of the proposed LTC-PC, and (c) amplitude of the co-polarized and cross-polarized reflected waves with $a=88 \mu \mathrm{m}$, $W=7 \mu \mathrm{m}, S=5 \mu \mathrm{m}$, and $L=45 \mu \mathrm{m}$.

As shown in Figure 2(a), co-axial strips only reflect co-polarized wave without generating crosspolarized wave [18]. The crossed strips, however, produce both co-polarized and cross-polarized reflected waves. The angle between the two crossed strips plays an important role in the amplitude difference of the two reflected waves, as shown in Figure 2(f). When the angle is close to $90^{\circ}$, the co-polarized and crosspolarized wave amplitudes will be equal and thus, we will have a more ideal circular polarized wave.

A asymmetric-diagonal structure is essential to achieve the best performance of LTC-PC. For this purpose, we added extra tails of gold, $d$, to the crossed strips, as depicted in Figure 1(b), to achieve higher asymmetry, which resulted in the extension of the bandwidth. Moreover, decreasing the distance of the two adjacent gold strips, $2 S$, increased the coupled electric field between them. As a result, the bandwidth operation of the LTC-PC increased. $2 S$ decreases by increasing the width of gold strips, $W$, toward the edge. Figure 3(a) and (b) show the electric field distribution for two different values of $2 S$ and $W$ at $1 \mathrm{THz}$. Reducing $2 S$, the capacitors between two adjacent strips increase, which leads to increasing electric field. Therefore, a frequency red-shift will happen in the first resonance frequency, resulting in a wider bandwidth. The variation of bandwidth versus distance between adjacent strips, $2 S$, is demonstrated 


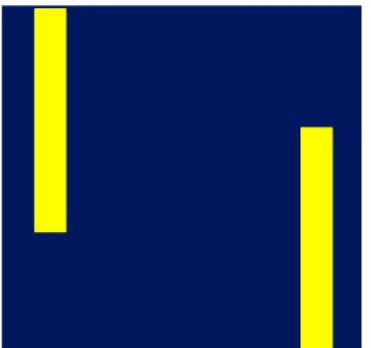

(a)

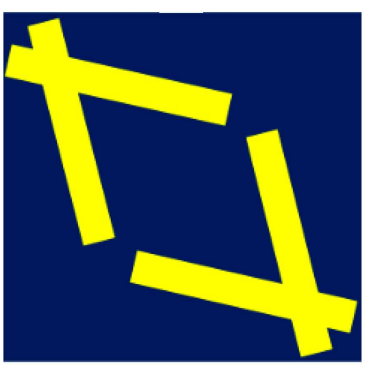

(c)

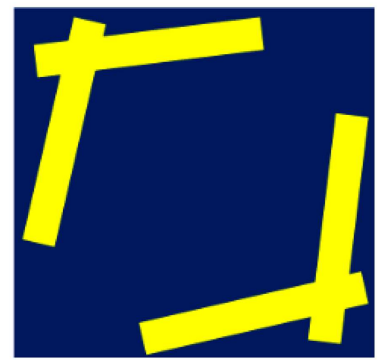

(e)

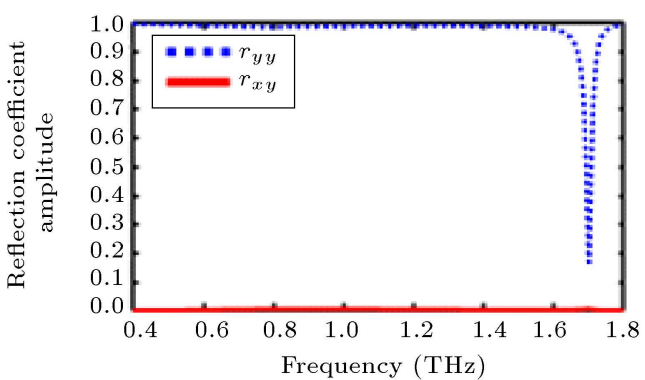

(b)
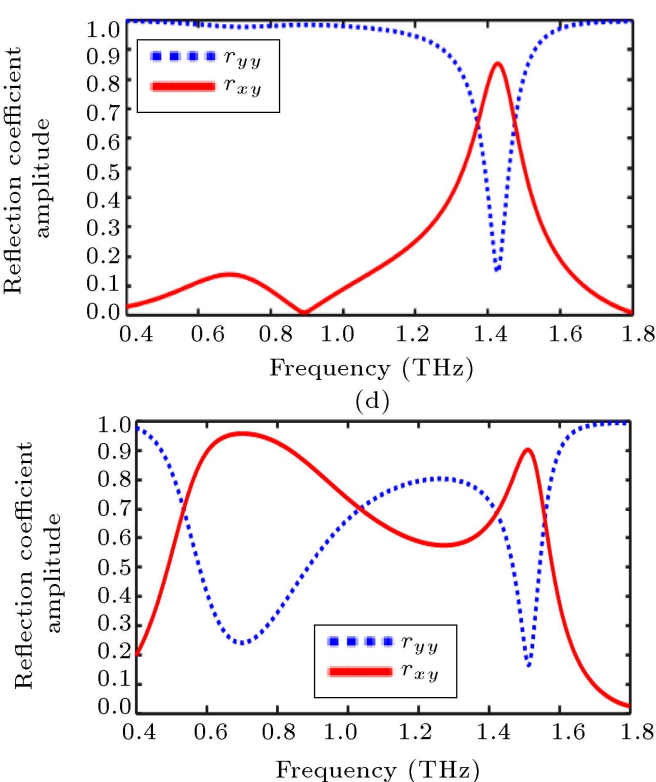

(f)

Figure 2. Unit cell of (a) the single strip and two crossed strips with angles (c) less than and (e) more than $90^{\circ} ;(\mathrm{b}),(\mathrm{d})$, and (f) amplitudes of the co-polarized and cross-polarized reflected waves for (a), (c), and (e), respectively.

in Figure 3(c). There is an optimum distance, which is considered $10 \mu \mathrm{m}$ for our proposed structure. According to the definition of bandwidth in LTC-PC, circular polarization occurs when the amplitudes of $r_{x y}$ and $r_{y y}$ are between $0.45-0.9$. When $2 S=10 \mu \mathrm{m}$, amplitudes of the polarization coefficients are on the edge of the bandwidth boundaries, thus the widest bandwidth is attained. For distances smaller than $10 \mu \mathrm{m}$, the amplitude of the first resonance is less than 0.45 , which leads to a narrower bandwidth. Besides, the effect of the extra tail of the gold strip, $d$, on the bandwidth is demonstrated in Figure 3(d). By increasing $d$, the inductance of each strip increases, which results in decreasing resonant frequency and consequently, increasing bandwidth. The optimum length for achieving the widest bandwidth is $d=S$.

Boundary conditions are periodic in $x$ and $y$ directions and non-reflective in the $z$-direction. The tetrahedral mesh structure with a minimum mesh size of $0.2 \mu \mathrm{m}$ is used for better accuracy in the $\mathrm{THz}$ regime. The structure is illuminated by a normal incident TE wave, which can be expressed as $E_{i}=a_{y} E_{y i} \cos (w t-$ $k z)$, and the reflected wave is:

$$
\begin{aligned}
E_{r}= & a_{x} E_{x r}+a_{y} E_{y r}=a_{x} E_{y i} r_{x y} \cos \left(w t-k z+\phi_{x y}\right) \\
& +a_{y} E_{y i} r_{y y} \cos \left(w t-k z+\phi_{y y}\right),
\end{aligned}
$$

where $r_{x y}=E_{x r} / E_{y i}, r_{y y}=E_{y r} / E_{y i}, \phi_{x y}$, and $\phi_{y y}$ represent the reflection coefficient magnitudes for the $y$-to- $x$ and $y$-to- $y$ polarization conversions and their corresponding angles, respectively. Circular polarization occurs when $r_{x y}=r_{y y}$ and $\Delta \varphi=\varphi_{y y}-\varphi_{x y}=$ $(2 n \pm 1 / 2) \pi$ for an integer value of $n$. The positive and negative signs represent the RHCP and LHCP, respectively $[11,19]$. By illuminating an incident TM wave, this behavior is inverted. Elliptically polarized wave is approximately considered as a circularly polarized wave, because a perfect circular polarization is not achievable practically. By using Stokes parameters in free space, the normalized Stokes parameters of the reflected wave can be expressed as $[11,20]$ :

$$
\begin{aligned}
& I_{n}=\left|r_{x y}\right|^{2}+\left|r_{y y}\right|^{2}, \\
& Q_{n}=\left|r_{y y}\right|^{2}-\left|r_{x y}\right|^{2}, \\
& U_{n}=2\left|r_{x y}\right|\left|r_{y y}\right| \cos \Delta \varphi,
\end{aligned}
$$




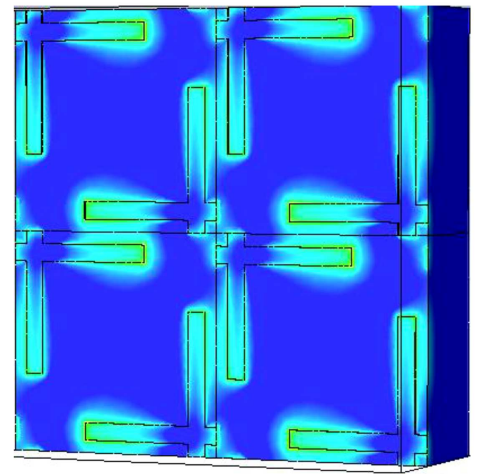

(a)

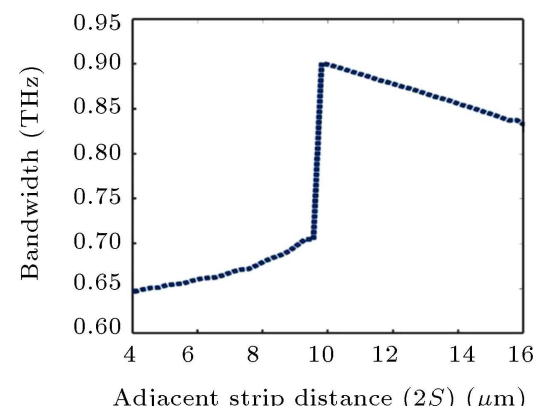

(c)
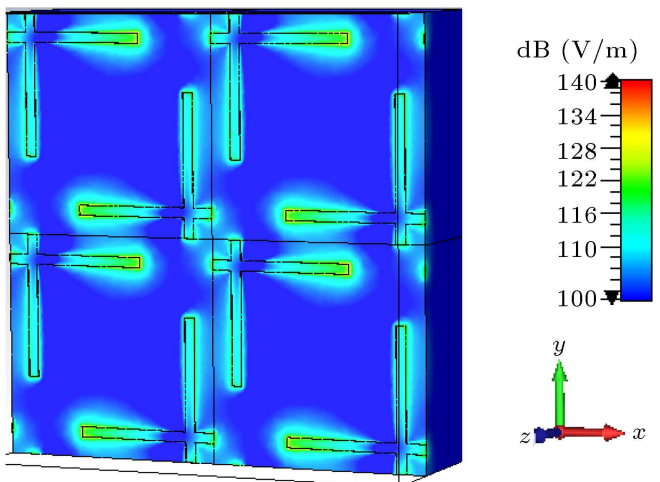

(b)

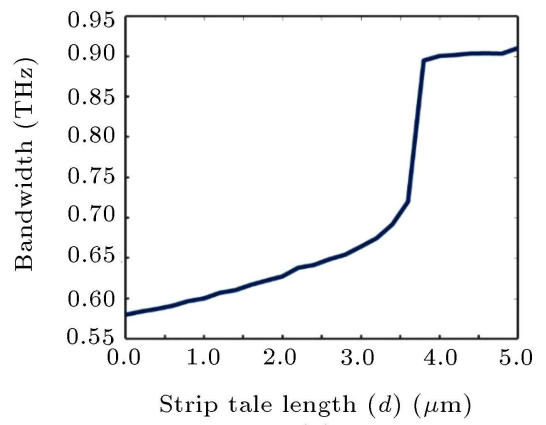

(d)

Figure 3. Distribution of the electric field in our proposed structure with (a) $S=8 \mu \mathrm{m}$ and $W=4 \mu \mathrm{m}$ and (b) $S=5 \mu \mathrm{m}$ and $W=7 \mu \mathrm{m}$. Amplitude of bandwidth for different (c) distances of adjacent strips and (d) lengths of strip tails.

$$
\begin{aligned}
& V_{n}=2\left|r_{x y}\right|\left|r_{y y}\right| \sin \Delta \varphi, \\
& I_{n}^{2}=Q_{n}^{2}+U_{n}^{2}+V_{n}^{2},
\end{aligned}
$$

where $I_{n}, Q_{n}, U_{n}$, and $V_{n}$ are total reflection, horizontal or vertical linear polarization, linear +45 or -45 polarization, and circular polarization of the reflected wave, respectively. These four Stokes parameters describe the wide-band waves and their polarizations. For a perfect circular polarization, $Q_{n}$ and $U_{n}$, which are linearly polarized waves, should be equal to zero [20].

According to the definitions of the Stokes parameters, the mathematical equation of ellipticity can be defined as the ratio $V_{n} / I_{n}$. In special cases, where
$V_{n} / I_{n}=-1$, the reflected wave is RHCP. On the other hand, when $V_{n} / I_{n}=+1$, the reflected wave is LHCP [18]. $A R$ is used for evaluating the circular polarization characteristic, which can be defined as $A R=10 \log (\tan b) . \beta$ is the ellipticity angle representing the shape of the ellipse, which can be calculated by $\beta=1 / 2 \cdot \sin ^{-1}\left(V_{n} / I_{n}\right)$. When the value of $\mathrm{AR}$ is less than $3 \mathrm{~dB}$, the reflected wave is approximately considered as a circularly polarized wave [21].

\section{Results and discussion}

As shown in Figure 4, within the bandwidth of $0.5-$ $1.41 \mathrm{THz}$ and $1.45-1.55 \mathrm{THz}$, the magnitudes of $r_{x y}$

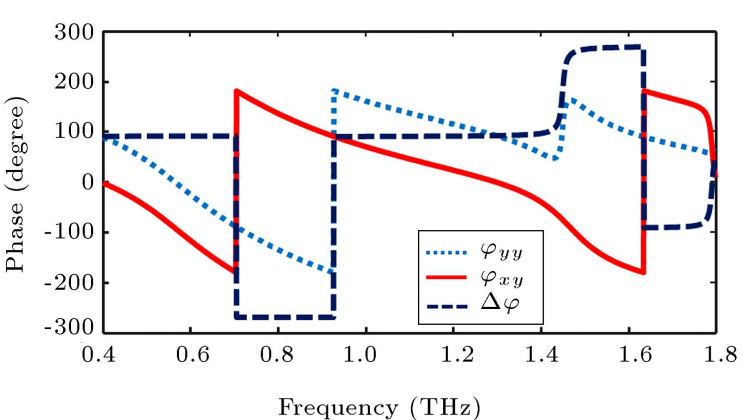

(b)

Figure 4. The $x$ - and $y$-components of the reflection coefficients of the structure in Figure 1: (a) Amplitude and (b) phases and phase differences. The dotted-dashed lines show the narrow- and wide-band bandwidths. 
and $r_{y y}$ are approximately the same and the difference of $\varphi_{x y}$ and $\varphi_{y y}$ is $90^{\circ}$ or $-270^{\circ}$ in the first frequency band and $-90^{\circ}$ or $270^{\circ}$ in the second one. The equal amplitudes and $\pm 90^{\circ}$ phase differences show that the reflected wave is a circularly polarized wave. According to Figure 5 and as per the Stokes parameters, in the range of 0.5 to $1.41 \mathrm{THz}$, the ellipticity is close to +1 and the reflected wave is LHCP. In the range of 1.45$1.55 \mathrm{THz}$, there is also an approximate ellipticity of -1 and an RHCP reflected wave. Also, the performance of the LTC-PC remains good even when illuminated by an oblique incident wave. It is also interesting that for less than 15 degrees of deviation from normal incident wave, the bandwidth of $\mathrm{PC}$ remains approximately the same and hence, the structure has a very low sensitivity to the incident wave angle. However, at higher angles, the bandwidth decreases.

The $A R$ spectrum of the output wave of the optimized structure in Figure 1 is depicted in Figure 6. The proposed LTC-PC operates satisfyingly and converts the polarization from linear to circular in the range of 0.5 to $1.41 \mathrm{THz}$ and 1.45 to $1.55 \mathrm{THz}$, because of the $A R$ value of less than $3 \mathrm{~dB}$. LHCP works in a wide range of frequencies and the RHCP works in a narrower range. The bandwidths of LHCP and RHCP increase by up to $15 \%$ and $66 \%$ in comparison with results reported in [11], respectively.

The thickness of the substrate and the length of

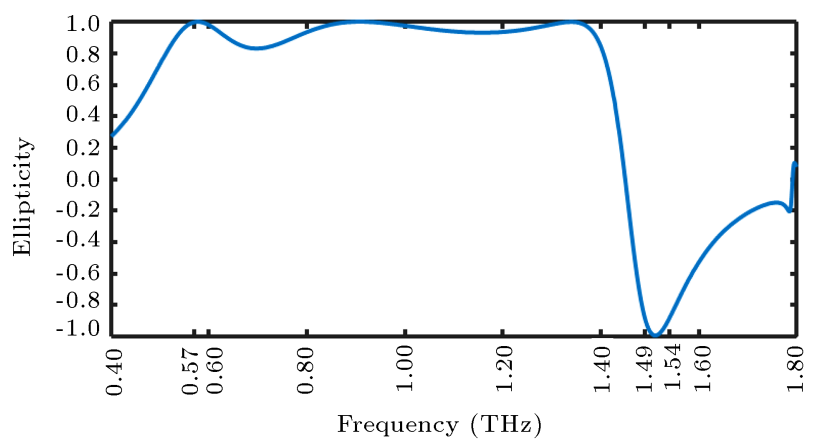

Figure 5. The ellipticity of the output wave of the structure in Figure 1, which is excited by a $y$-polarized plane wave.

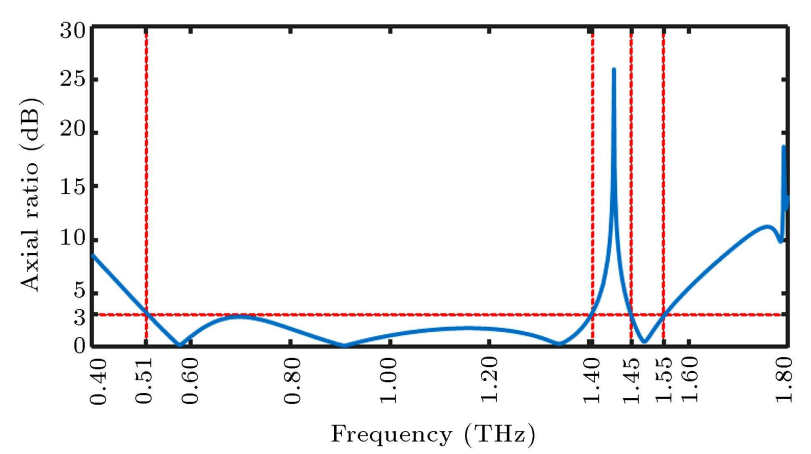

Figure 6. Axial ratio of the output wave of the Linear To Circular Polarization Converter (LTC-PC) in Figure 1.
Table 1. Comparison of the efficiency and bandwidth of the proposed structure with some earlier similar reports.

\begin{tabular}{ccc}
\hline Reference & Efficiency & Bandwidth \\
\hline$[22]$ & $>95 \%$ & $7.5-11.9 \mathrm{GHz}$ \\
{$[23]$} & High & $9.38-20.36 \mathrm{GHz}$ \\
{$[11]$} & $>85 \%$ & $0.6-1.4 \mathrm{THz}$ \\
This work & $>95 \%$ & $0.5-1.31 \mathrm{THz}$ \\
\hline
\end{tabular}

the arm have significant effects on the results. The magnitude spectra of the reflection coefficients and the $A R$ s for three different values of structure substrate thickness, $h$, and the length of the arm, $L$, are, respectively, illustrated in Figures $7(\mathrm{a}),(\mathrm{b})$, and 8(a), (b). The optimum values of $h$ and $L$ are $47 \mu \mathrm{m}$ and $55 \mu \mathrm{m}$, respectively.

The energy conversion efficiency $(\eta)$ is also calculated by the ratio of output wave energy to the incident wave energy. The normalization of the Stokes parameters lead to equal values of $I_{n}$ and $\eta$. The spectrum for the energy conversion efficiency of the proposed device is illustrated in Figure 9. $\eta$ is more than $89 \%$ for the LHCP and more than $85 \%$ for the RHCP. The efficiency of the LHCP wave is more than $95 \%$ in $80 \%$ of the bandwidth. The results indicate the high efficiency of our proposed structure. The efficiency and bandwidth of our proposed LTC-PC are compared with three other similar works in Table 1.

\section{Conclusion}

In this study, a high-efficiency metasurface-based wideband linear-to-circular polarization converter (LTCPC) was proposed in the terahertz frequency range. It was shown that when the angle between two crossed strips was close to $90^{\circ}$, the reflection wave was closer to circularly polarized. Furthermore, different parameters of the structure such as thickness of the substrate $(h)$, gold strips length $(L)$, distance between two adjacent gold strips $(2 S)$, and length of extra tails $(d)$ were optimized to gain the highest efficiency and widest bandwidth. Efficiency of the proposed structure was more than $89 \%$ within an ultra-wideband frequency range of $0.5-1.41 \mathrm{THz}$. Moreover, the reflected wave was left-handed circularly polarized if a linear TE polarization input wave was applied. The efficiency of the conversion was more than $95 \%$ in $80 \%$ of this bandwidth. Within the frequency range of $1.45-$ $1.55 \mathrm{THz}$, the efficiency was more than $85 \%$ and the reflected wave was right-handed circularly polarized. The LTC converter has potential applications in electromagnetic measurement, antenna design, and cloaking technology. 


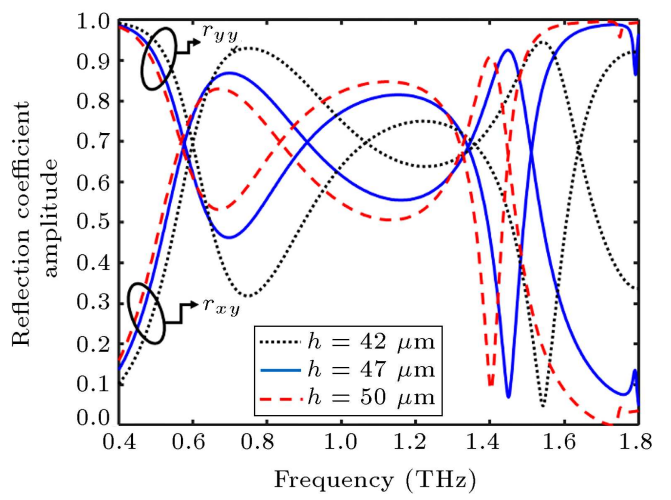

(a)

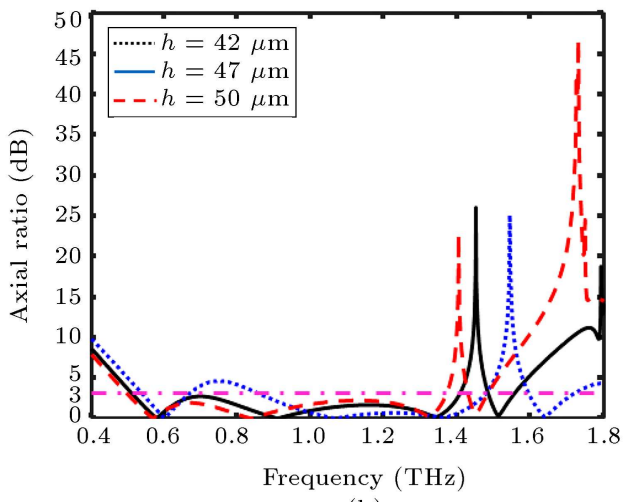

(b)

Figure 7. Spectra of (a) the reflection coefficient amplitude and (b) Axial Ratio (AR) of the output wave for three different thicknesses of the structure substrate.

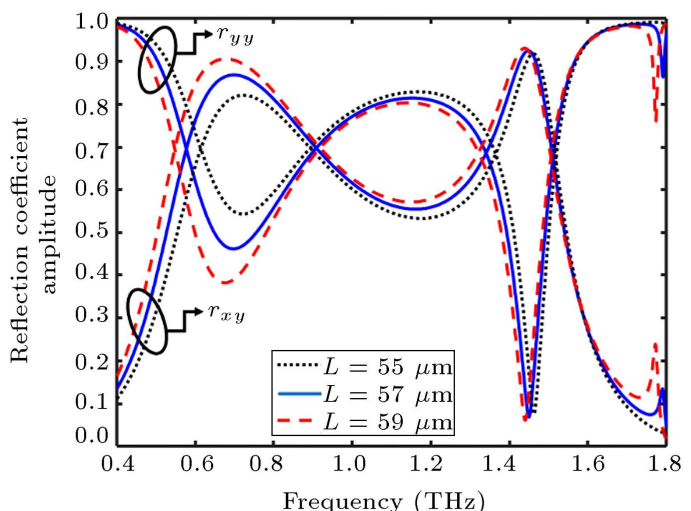

(a)

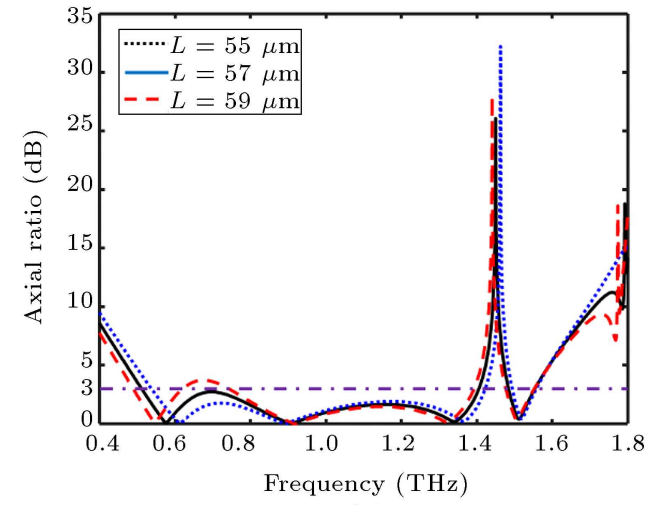

(b)

Figure 8. Spectra of (a) the reflection coefficient amplitude and (b) Axial Ratio (AR) of the output wave for three different strip lengths.

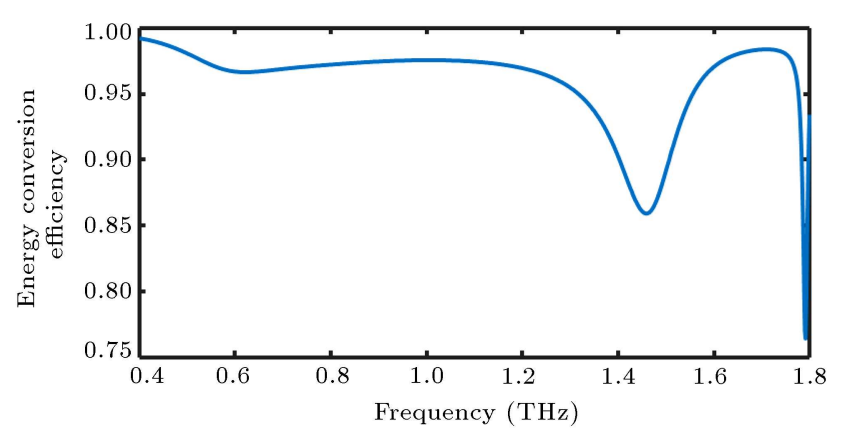

Figure 9. Spectrum of the energy conversion efficiency $(\eta)$ of the proposed Linear To Circular Polarization Converter (LTC-PC) in Figure 1.

\section{References}

1. Chen, H.T., Taylor, A.J., and Yu, N. "A review of metasurfaces: physics and applications", Reports on Progress in Physics, 79(7), p. 076401 (2016).

2. Mendhe, S.E. and Kosta, Y.P. "Metamaterial properties and applications", International Journal of Information Technology and Knowledge Management, 4(1), pp. 85-89 (2011).
3. Achouri, K. and Caloz, C. "Design, concepts, and applications of electromagnetic metasurfaces", Nanophotonics, 7(6), pp. 1095-1116 (2018).

4. Glybovski, S.B., Tretyakov, S.A., Belov, P.A., et al. "Metasurfaces: From microwaves to visible", Physics Reports, 634, pp. 1-72 (2016).

5. Achouri, K., Khan, B.A., Gupta, S., et al. "Synthesis of electromagnetic metasurfaces: principles and illustrations", arXiv Preprint arXiv: 1510.05997 (2015).

6. Sarychev, A.K., Lagarkov, A.N., Ivanov, A.V., et al. "Metal-dielectric resonances in tip silicon metasurface and SERS based nanosensors", In Plasmonics: Design, Materials, Fabrication, Characterization, and Applications $X V$, 10346, p. 103460C (2017).

7. Sleasman, T., Imani, M.F., Boyarsky, M., et al. "Reconfigurable metasurface aperture for security screening and microwave imaging", In Passive and Active Millimeter-Wave Imaging XX, 10189, p. 101890G (2017).

8. Liu, Z. and Bai, B. "Ultra-thin and high-efficiency graphene metasurface for tunable terahertz wave manipulation", Optics Express, 25(8), pp. 8584-8592 (2017). 
9. Yachmenev, A.E., Lavrukhin, D.V., Glinskiy, I.A., et al. "Metallic and dielectric metasurfaces in photoconductive terahertz devices", A Review. Optical Engineering, 59(6), p. 061608 (2019).

10. Gao, X., Han, X., Cao, W.P., et al., "Ultrawideband and high-efficiency linear polarization converter based on double V-shaped metasurface", IEEE Transactions on Antennas and Propagation, 63(8), pp. 3522-3530 (2015).

11. Jiang, Y., Wang, L., Wang, J., et al. "Ultra-wideband high-efficiency reflective linear-to-circular polarization converter based on metasurface at terahertz frequencies", Optics Express, 25(22), pp. 27616-27623 (2017).

12. Gao, X., Yang, W., Cao, W., et al. "Bandwidth broadening of a graphene-based circular polarization converter by phase compensation", Optics Express, 25(20), pp. 23945-23954 (2017).

13. Yang, Z., Yu, S., Kou, N., et al. "Ultrathin tri-band reflective cross-polarization artificial electromagnetic metasurface", Journal of Electromagnetic Waves and Applications, pp. 1-11 (2020).

14. Chen, H.T. and Chang, C.C. "Broadband terahertz polarization conversion using metasurfaces", (Conference Presentation), In Terahertz Emitters, Receivers, and Applications IX, 10756, p. 107560C (2018).

15. Swain, R., Chatterjee, A., Nanda, S., et al. "A linear-to-circular polarization conversion metasurface based wideband aperture coupled antenna", Journal of Electrical Engineering \& Technology, 15, pp. 12931299 (2020).

16. Lin, B.Q., Lv, L., Guo, J., et al. "Ultra-wideband circular polarization-maintaining reflection realized by an anisotropic metasurface", Journal of Electromagnetic Waves and Applications, 34(10), pp. 1420-1429 (2020).

17. Khan, B., Kamal, B., Ullah, S., et al. "Design and experimental analysis of dual-band polarization converting metasurface for microwave applications", Scientific Reports, 10(1), pp. 1-13 (2020).

18. Zhang, L., Zhou, P., Lu, H., et al. "Realization of broadband reflective polarization converter using asymmetric cross-shaped resonator", Optical Materials Express, 6(4), pp. 1393-1404 (2016).

19. Cong, L., Cao, W., Zhang, X., et al. "A perfect metamaterial polarization rotator", Applied Physics Letters, 103(17), p. 171107 (2013).

20. Goldstein, D., Polarized Light, pp. 59-61, CRC Press (Dec. 16, 2016).

21. Li, Y., Zhang, J., Qu, S., et al. "Achieving wideband linear-to-circular polarization conversion using ultra-thin bi-layered metasurfaces", Journal of Applied Physics, 117(4), p. 044501 (2015).

22. Khan, M.I., Khalid, Z., and Tahir, F.A. "Linear and circular-polarization conversion in X-band using anisotropic metasurface", Scientific Reports, 9(1), pp. 1-11 (2019).

23. Fu, C., Sun, Z., Han, L., et al. "High-efficiency "dual-frequency reflective linear polarization converter based on metasurface for microwave bands", Applied Sciences, 9(9), p. 1910 (2019).

\section{Biographies}

Mahsa Barkabian received her BSc in Electrical Engineering-Telecommunications in 2017 from Zanjan University, Zanjan, Iran. In 2017, she started her MSc in Telecommunications-Fields and Waves at $\mathrm{K}$. N. Toosi University of Technology, Tehran, Iran. She worked in Optical Communication Lab under the supervision of Prof. Granpayeh in the field of 2D materials, metasurfaces, graphene, terahertz technology, and polarization conversion.

Narges Dalvand in 2016 received her BSc degree in Electronics Engineering from Shahid Chamran University, Khousestan, Ahvaz, Iran. In 2017, she joined the Electrical Department of K. N. Toosi University of Technology, Tehran, Iran as an MSc student under the supervision of Dr. Zandi. Her research interests include terahertz sources, graphene and complex materials, nonlinear photonics, and optical communication.

Hesam Zandi received BSc, MSc, and PhD degrees in Electrical Engineering from Sharif University of Technology in 2005, 2007, and 2012, respectively. He conducted research on the development of a quantum bit device, which led to double entangled qubits having high fidelity reading process at SERL. He got a postdoc research fellowship from SUT, where he developed an RSFQL structure for ultra-high speed digital circuits. In 2016, he joined K. N. Toosi University of Technology, where he is currently an Assistant Professor in Electrical Engineering. His research interests are mainly focused on the design, modelling, and fabrication of $\mathrm{THz}$ devices and systems; metal-oxide sensors; and superconducting devices. He has recently been chosen as the head of the EML laboratory which is one the well-known establishments at KNTU.

Nosrat Granpayeh received his BSc, MSc, and PhD degrees in Telecommunication Engineering from the Telecommunication College, Radio and Television College in Tehran, Iran, and University of NSW, Sydney, Australia, in 1975, 1980, and 1996, respectively. In 1975, as an honor graduate of the Faculty of Electrical Engineering of K. N. Toosi University of Technology, Tehran, Iran, he was employed as an instructor. He was promoted to Lecturer, Assistant Professor, Associate Professor, and Professor, respectively, in 1980, 1996, 2007, and 2016. His research interests are in optical 
devices, equipment, and materials; optical fibers; and optical fiber effects. He is the author and co-author of 100 peer reviewed journal and 100 conference papers. Professor Granpayeh is a senior member of IEEE, senior member of the Optical Society of America
(OSA), member of the Board of Directors of Optics and Photonics Society of Iran (OPSI), member of the Iran Society of Engineering Education (ISEE), and the Chair of the Professional Activities Committee of IEEE Iran Section. 